\title{
Análisis del costo financiero en una empresa PYMES, durante el período 2014
} $-2016$

\section{Analysis of the financial cost in a PYMES company, during the period 2014 - 2016}

Galo Mauricio Durán Salazar

Omar Daniel Apolinario Zatizabal

Ana Benítez Morán

María Lisbeth Carrera Gómez

Universidad de Guayaquil, Ecuador

Autor por correspondencia: galo.duransa@ug.edu.ec, omar.apolinarioza@ug.edu.ec mariang.benitez.m@gmail.com,marilizc.gomez@hotmail.com

Fecha de recepción: 01 de agosto del 2019 - Fecha de aceptación: 14 de octubre del 2019

\section{Resumen}

La presente investigación tuvo por objetivo, analizar el costo financiero en el que incurrió una Pymes durante los años 2014-2015-2016, revisando indicadores financieros y estimando la tasa de interés de los préstamos bancarios obtenidos, para luego comparar con otras formas de financiamiento que tienen las empresas para conseguir recursos. Se analizó dos teorías sobre las formas que tienen las empresas de conseguir recursos financieros; una teoría es sobre Pecking order que indica 3 formas de obtener endeudamiento a través de las utilidades del ejercicio, préstamos bancarios y accionistas; la otra teoría se denomina Trade Off expresa que los gastos financieros, generan ahorro tributario. El análisis de datos estimó que la tasa de interés pagada por la empresa fue del 18\%, relacionándose con lo que sucede con el mercado, donde las PYMES obtienen préstamos con tasas de hasta el $22 \%$ y las decisiones tomadas para endeudarse siguen el esquema de las teorías revisadas. Se concluye que una de las formas de endeudamiento es el uso del mercado de valores que ofrece tasas de interés bajas, a través de la denomina RED.

Palabras Clave: tasas de interés; costo financiero; préstamos bancarios; caso de estudio

\begin{abstract}
The objective of this research was to analyze the financial cost incurred by SMEs during the years 2014-2015-2016, reviewing financial indicators and estimating the interest rate on bank loans obtained, and then comparing them with other forms of financing that companies have to obtain resources. Two theories were analyzed on the ways that companies have to obtain financial resources; one theory is about Pecking order that indicates three ways to obtain indebtedness through the profits of the year, bank and shareholder loans; The other theory is called Trade Off, indicated that financial expenses generate tax savings. The data analysis
\end{abstract}


estimated that the interest rate paid by the company was $18 \%$, relating to what happens with the market, where SMEs obtain loans with rates over $22 \%$ and the decisions taken to get money are according to the revised theories. It is concluded that one of the forms of indebtedness is the use of the stock market that offers low interest rates, through the name RED.

Key words: interest rate; financial cost; bank loans; case study

\section{Introducción}

En el plano mundial las pequeñas y medianas empresas PYMES se consideran a partir de ciertos parámetros como número de empleados, nivel de ventas y total de activos; están inmersos en diferentes sectores de la economía, como son servicios, comercialización, industria entre otros. Según OMPI (2015), el 90\% de las empresas son PYMES en el mundo y contribuyen a la generación de empleo y desarrollo de la economía.

La segmentación de los mercados financieros y la discriminación negativa hacia las pequeñas y medianas empresas son aspectos que prevalecen en las economías latinoamericanas. La aplicación de diferenciales en las tasas de interés de los préstamos según el tamaño de empresa, la escasa participación de las pymes en el crédito al sector, privado y la amplia utilización de proveedores y autofinanciamiento para obtener capital de giro o llevar a cabo inversiones, son elementos que denotan la existencia de dificultades de acceso para este tipo de agentes (Ferraro, Goldstein, Zuleta, \& Garrido, 2011).

De acuerdo a Lecuona (2009) los bancos mexicanos identificaron las causas por las cuales restringen préstamos a las PYMES, entre las que se puede mencionar:

- Alta mortalidad que existe en las PYMES.

- Información poco confiable de las empresas.

- Costo de monitoreo elevado, por el seguimiento que debe realizar el banco a su cliente, en vista del "Daño moral", el cual se presenta cuando el empresario usa recursos del negocio en sus finanzas personales.

- Informalidad en el negocio y su escaza profesionalización.

El Banco Mundial (2014) confirmó las limitaciones que tienen estas empresas para acceder a crédito del sistema financiero, basados en la escasa garantía que tienen y la contratación inadecuada de profesionales para administrar el negocio, lo cual genera que estos emprendimientos desaparezcan a mediano plazo, esto provoca que los bancos les cobren altas tasas de interés por préstamos, por la incertidumbre que existe, en que las PYMES puedan cumplir con sus obligaciones financieras futuras.

Por otro lado, Osano y Languitone (2016) indicaron que exigir a las PYMES garantías de los socios para acceder a créditos desmotivan a estas empresas, además es una forma de discriminación; al final los estados financieros de dichas empresas no representan la situación real del negocio ya que no son auditados.

Según el Global Entrepreneurship Monitor (GEM) las PYMES se caracterizan por su elevada tasa de mortalidad, en el caso de Colombia en el año 2009 cerraron el 16\% de estas 
empresas en el corto plazo, lo cual no garantiza que puedan consolidarse a largo plazo (Romero, Zuray, \& Mary, 2015).

Londoño \& Sanchez (2017) mencionó que existe poca bancarización hacia las PYMES, por ello, estas empresas se financian con la utilidades acumuladas o préstamos de socios, para poder operar y cubrir capital de trabajo o compra de activos fijos.

En el Ecuador, la Superintendencia de Compañías es el ente público que regula a las sociedades, estableció los tipos de empresas en 4 categorías, lo cual, se puede observar en la tabla 1, mostrando la clasificación de compañías que existe en el país, resaltado en negrilla las pequeñas.

Tabla. 1

Clasificación de las empresas por su tamaño en el marco ecuatoriano

\begin{tabular}{lcccc}
\hline \multicolumn{1}{c}{ Variable } & Micro & Pequeña & Mediana & Grande \\
\hline Personal Ocupado & 1 a 9 & 10 a 49 & 50 a 199 & Mayor a 200 \\
& & & $1^{\prime} 000.001$ a & Mayor \\
Ventas (anual) & Hasta a 100.000 & 100.001 a 1'000.000 & $5^{\prime} 000.000$ & $5^{\prime} 000.001$ \\
& & & 750.001 a & \\
Monto de activos & Hasta a 100.000 & 100.001 a 750.000 & 3'999.999 & Mayor 4'000.000 \\
\hline
\end{tabular}

Fuente: Elaboración propia

La situación de las PYMES en Ecuador, según el Ministerio de Industrias y Productividad (2012), indicó que son el recurso de riqueza que aporta mucho al país, siendo sus características tener de 10 a 49 trabajadores, un valor de ventas o ingresos brutos anuales entre cien mil uno y un millón USD 100.001 -USD 1'000.000, dado aquello, significa que por cada pequeña o mediana empresa creada, el Estado recauda una cantidad determinada en tributos; por lo anterior, se puede entender la importancia de este grupo de empresas para la economía.

De acuerdo a las Superintendencia de Compañías (2016) la mayor porción de sociedades, está compuesta por las microempresas cuyo segmento obtuvo el $51.30 \%$ de entre el total de empresas, registradas en la Superintendencia hasta el año 2016, lo cual se aprecia en la figura 1.

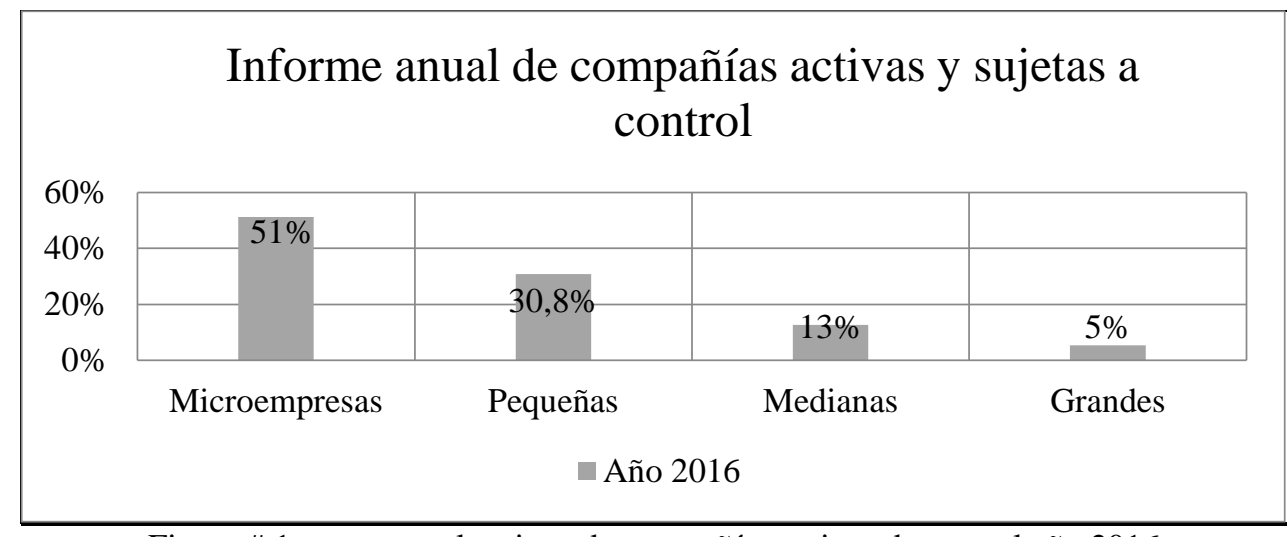

Figura \# 1 se muestra los tipos de compañías activas durante el año 2016 
Respecto al financiamiento que recibe una empresa grande, es diferente al que obtiene una PYMES, tal como se aprecia en la figura 2, considerando que las pequeñas empresas, la tasa de interés que pagaron fue de hasta el $22 \%$, frente a un $12 \%$ que se aplicó para las grandes empresas, lo cual denota, el alto costo financiero con el que incurren las PYMES. Una de las causas por la alta tasa, es la falta de garantías que respalden los préstamos, ya que una PYMES, normalmente sus activos son pequeños o no poseen bienes que respaldes a los créditos.

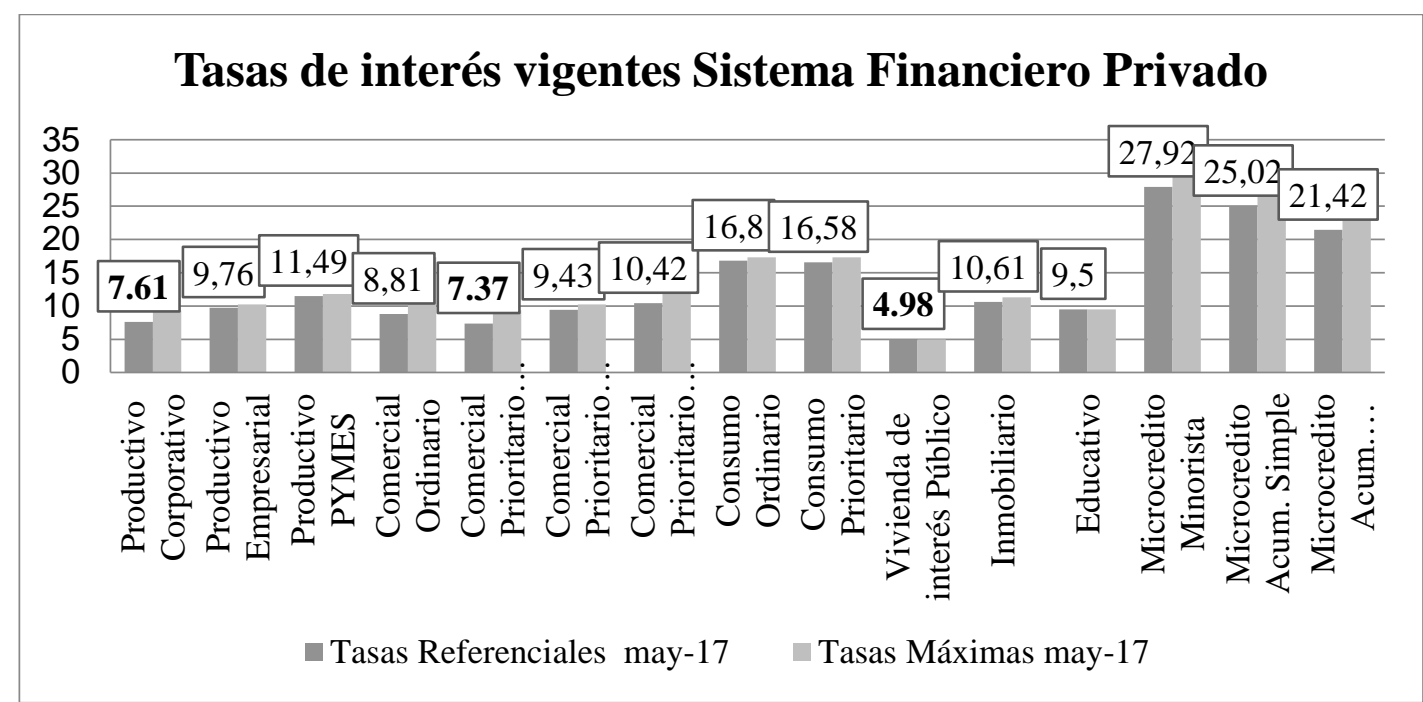

Figura \# 2 se puede observar las diferentes tasas que se aplican a diferentes tipos de negocio en el Ecuador, aplicadas hasta mayo del año 2017.

La presente investigación analizó una empresa PYMES, ubicada en la ciudad de Guayaquil, siendo su objetivo "Analizar el costo financiero en el que incurrió la empresa durante los años 2014-2015-2016”, para ello, se aplicó el uso de indicadores financieros y se comparó la tasa de interés que pagó la empresa, con otras opciones que existen en el mercado. En base a lo anterior, se denominó a la empresa, "Caso de estudio"; esta compañía durante el período 20142016 tuvo menos de 40 empleados y sus ventas anuales superaron el millón de dólares, se dedicó a la comercialización de sabores artificiales para las comidas.

\section{Problemática}

La figura 3 indica la problemática de la empresa, que hace referencia a la falta de liquidez, explicada por causas como la ausencia de planeación financiera y control de la cartera, lo que provocó retraso en el cumplimiento de sus obligaciones con sus proveedores y organismo de control, por ello, acudió al sistema financiero a solicitar crédito, pero en base a lo indicado anteriormente, las PYMES obtienen crédito a una tasa de interés de hasta el $22 \%$ de costo financiero, lo cual afectó de manera importante a los flujos de efectivo del negocio. 


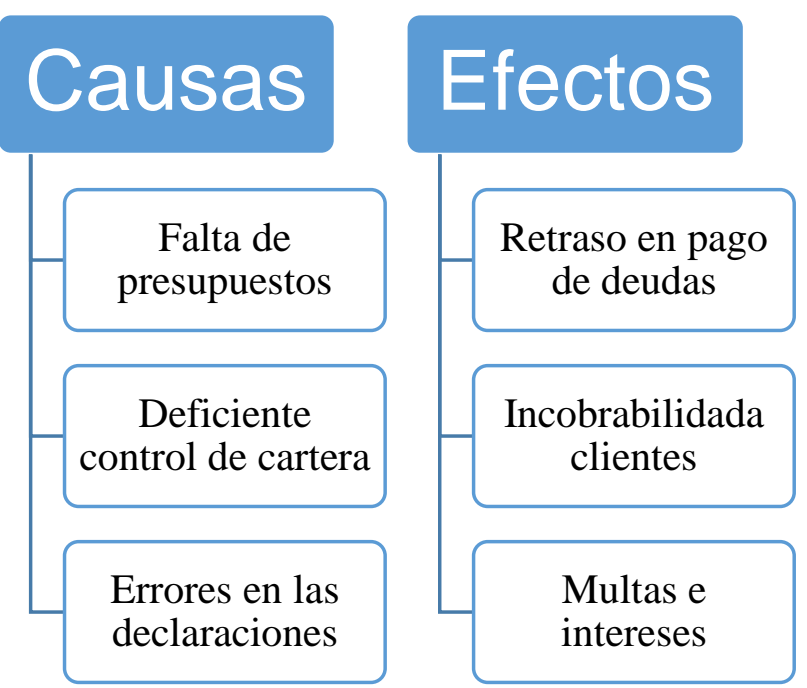

Figura \# 3. Se presenta las causas y efectos de la problemática de liquidez en la empresa "Caso de estudio"

\section{Marco teórico}

Dentro de la revisión de conceptos y definiciones sobre endeudamiento o financiamiento de empresas, se encontraron dos teorías, sobre las cuales se referenció, si lo actuado por la empresa responde a dichos enfoques teóricos. Los dos temas revisados se resumen a continuación:

- Teoría de Jerarquización financiera denominada Pecking order, según Azofra \& Fernández (1999), expresaron básicamente que las empresas buscan financiamiento en primer lugar con los socios, es decir, fondos internos, luego utilizando al sistema financiero y finalmente con deuda pública.

- Teoría de Apalancamiento objetivo, denominado Trade Off, según Murray \& Vidhan (2003) indicaron que las empresas que obtienen préstamos del sistema financiero, reducen el pago de impuestos, por los intereses cancelados, lo cual genera mayor rentabilidad para la sociedad.

\section{Metodología}

La metodología aplicada fue el "Caso de estudio", según Martínez (2006) indicó que este método es valioso en la investigación ya que mide y evalúa todas las situaciones involucradas en el fenómeno estudiado y los datos pueden obtenerse de manera cualitativa y cuantitativa. La investigación realizada tiene un enfoque cuantitativo, dado el análisis de datos usando información de los estados financieros de los períodos 2014 al 2016, tomados de la Superintendencia de Compañías, entidad que regula a las empresas en el Ecuador.

\section{Análisis de datos}

El Estado de Resultado de la empresa Caso de Estudio, información tomada de la Superintendencia de Compañías, se presenta en la tabla 2 de manera resumida, indicando los 
datos de los períodos analizados y sobre todo, el gasto de interés generado en los periodos fiscales; y la utilidad neta de cada período que representó entre el $1 \%$ y $2 \%$ de los ingresos del negocio.

Tabla.2

Estado de Resultados Integrales

\begin{tabular}{|c|c|c|c|c|c|c|}
\hline ESTADO RES ULTADOS & 2014 & $\%$ & 2015 & $\%$ & 2016 & $\%$ \\
\hline INGRESOS & 841,621 & $100 \%$ & 974,030 & $100 \%$ & $1,040,509$ & $100 \%$ \\
\hline COSTOS & 505,532 & $60 \%$ & 607,112 & $62 \%$ & 585,744 & $56 \%$ \\
\hline GASTOS & 293,733 & $35 \%$ & 339,831 & $35 \%$ & 406,294 & $39 \%$ \\
\hline FINANCIEROS & 8,410 & $1 \%$ & - & $0 \%$ & 19,250 & $2 \%$ \\
\hline UTILIDAD CONTABLE & 33,946 & $4 \%$ & 27,087 & $3 \%$ & 29,221 & $3 \%$ \\
\hline $15 \%$ TRABAJADORES & 5,092 & $1 \%$ & 4,063 & $0 \%$ & 4,383 & $0 \%$ \\
\hline \multirow[t]{2}{*}{ IMPUESTO A LA RENTA } & 12,585 & $1 \%$ & 10,895 & $1 \%$ & 11,448 & $1 \%$ \\
\hline & & & & $0 \%$ & & \\
\hline UTILIDAD NETA & 16,269 & $2 \%$ & 12,129 & $1 \%$ & 13,390 & $1 \%$ \\
\hline
\end{tabular}

Fuente: elaboración propia

El análisis financiero realizado a determinados indicadores, se presenta en la tabla 3, se puede observar de manera general, la empresa obtuvo ratios sin novedades, es decir, la compañía Caso de Estudio generó liquidez, sus endeudamientos eran manejables, cobró más rápido a sus clientes, que el tiempo que usó para cancelar a sus proveedores; y generó utilidades del 1\% promedio, para cada año, por lo que en base a los resultados, la empresa sin problemas pudo acceder a créditos del sistema financiero nacional.

Tabla.3

Resumen de ratios financieros

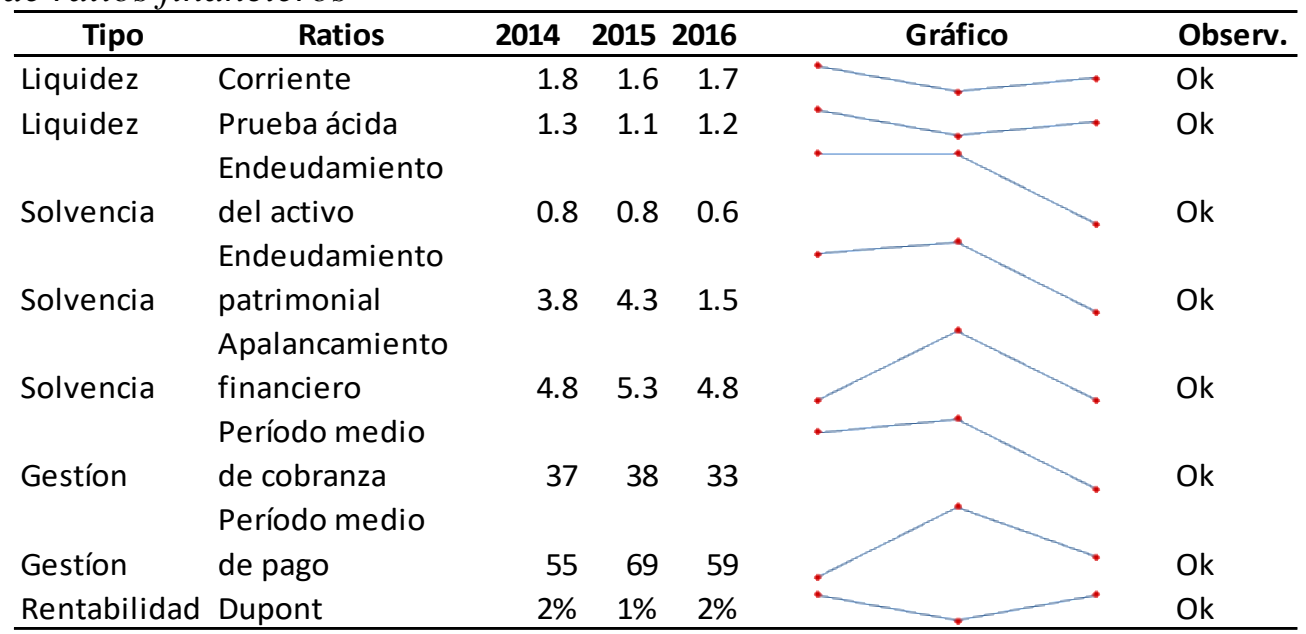

Fuente: elaboración propia 


\section{Análisis de los costos financieros}

De acuerdo a la revisión del Estado de Situación Financiera de la empresa Caso de Estudio, los préstamos bancarios realizados y gastos financieros reconocidos en el Estado de Resultados, para el período de estudio se presenta en la tabla 4.

Tabla.4

Detalle de préstamos bancarios

\begin{tabular}{rrrrr}
\hline Años & $\begin{array}{c}\text { Total } \\
\text { préstamo }\end{array}$ & $\begin{array}{c}\text { Porción } \\
\text { Corriente }\end{array}$ & $\begin{array}{c}\text { Porción No } \\
\text { corriente }\end{array}$ & $\begin{array}{c}\text { Gasto } \\
\text { Financiero }\end{array}$ \\
\hline 2014 & 90,695 & 60,879 & 29,816 & 8,410 \\
2015 & 195,695 & 76,578 & 119,117 & 11,659 \\
2016 & 155,443 & 75,220 & 80,223 & 17,167 \\
Total & $\mathbf{4 4 1 , 8 3 3}$ & $\mathbf{2 1 2 , 6 7 7}$ & $\mathbf{2 2 9 , 1 5 6}$ & $\mathbf{3 7 , 2 3 6}$ \\
\hline
\end{tabular}

Fuente: elaboración propia

Con los valores de los préstamos y los gastos financieros, se calculó la tasa de interés que pagó la empresa, obteniendo un resultado del 18\%, siendo una tasa alta y que coincide con lo planteado anteriormente sobre los costos financieros que pagan las PYMES en el sector bancario. La tasa del $18 \%$ se comparó con otras alternativas que tienen las PYMES, para financiarse, como es el aporte de socios, préstamos bancarios con instituciones públicas y la denomina REB que consiste con obtener fondo del mercado de valores; conforme lo define la Bolsa de Valores (2014) el REB es el registro especial bursátil constituye un mecanismo para la anotación y negociación de valores de renta fija, de tal manera que las sociedades puedan obtener recursos para sus operaciones.

Esta comparación se presenta en la tabla 5, donde se puede observar el ahorro en la tasa de interés que podría haber obtenido la empresa, siendo la más conveniente obtener recursos a través del mercado de valores, ya que se hubiera reducido el gasto financiero y como tal la salida de efectivo por los pagos al prestamista.

Tabla.5

Comparativo de tasas de fuentes de financiamiento en la empresa Caso de Estudio

\section{Tasa}

\begin{tabular}{lrcrr} 
Financiamiento & \multicolumn{1}{c}{ Tasa } & Indicador & Bancaria & \multicolumn{1}{c}{ Ahorro } \\
\hline CFN B.P. & $11.83 \%$ & $<$ & $18 \%$ & $6.2 \%$ \\
REB & $9.71 \%$ & $<$ & $18 \%$ & $8.3 \%$ \\
Socios & $10.19 \%$ & $<$ & $18 \%$ & $7.8 \%$ \\
\hline
\end{tabular}

Fuente: elaboración propia

\section{Contrastación teórica}

Como se planteó anteriormente, la empresa pagó altos intereses por sus préstamos bancarios, en base a las teorías revisadas previamente, la forma de obtención de financiamiento en la empresa Caso de Estudio, responde a las teorías de Pecking order y Trade Off. En la tabla 6 se indica la forma de financiarse que tiene una empresa basado en el modelo Pecking Order y el 
costo que representa, en base a ello, las estrategias de financiamiento en la empresa Caso de Estudio fue emisión de deuda con instituciones financiera a un costo del $18 \%$ y conforme lo indica la teoría su costo es elevado, esta decisión se tomó ya que al ver los estados financieros la utilidad neta del ejercicio era inferior a lo que la empresa necesitó para operar en los periodos 2014 al 2016.

\section{Tabla.6}

\section{Teoría del Pecking Order}

\begin{tabular}{ll}
\multicolumn{1}{c}{$\begin{array}{c}\text { Formas de } \\
\text { Financiamiento }\end{array}$} & $\begin{array}{c}\text { Teoría de los costos } \\
\text { de Transacción }\end{array}$ \\
\hline 1. Beneficios retenidos & Bajos costos \\
2. Emisión de deuda & Elevados costos \\
3. Emisión de acciones & Muy altos costos \\
\hline
\end{tabular}

Tomado de la Teoría del Pecking Order por Revista Actualidad Empresarial N 169 Segunda quincena octubre 2008

En cuanto a la teoría del Trade Off, indica que, al generarse costos financieros, se produce un ahorro tributario, equivalente al valor del gasto por la tarifa del impuesto a la renta, teóricamente se aplicó en la empresa y en la tabla 7 se presenta el impacto real en efectivo que tuvo la empresa en cada período. Por lo anterior, a pesar de haber realizado prestamos financieros a una tasa calculada del 18\%; este gasto redujo en pago del impuesto a la renta, siendo menor el impacto en materia de salida neto efectivo, conforme se muestra en la tabla 7.

Tabla.7

Comparación Gastos de Interés y ahorro tributario

\begin{tabular}{crrr}
\hline Año & $\begin{array}{c}\text { Gasto } \\
\text { Intereses }\end{array}$ & $\begin{array}{r}\text { Ahorro } \\
\text { tributario }\end{array}$ & $\begin{array}{r}\text { Salida neta } \\
\text { de efectivo }\end{array}$ \\
\hline 2014 & 8,410 & 2,102 & 6,307 \\
2015 & 11,659 & 2,915 & 8,744 \\
2016 & 17,167 & 4,292 & 12,875 \\
Total & $\mathbf{3 7 , 2 3 6}$ & $\mathbf{9 , 3 0 9}$ & $\mathbf{2 7 , 9 2 7}$ \\
\hline
\end{tabular}

Fuente: elaboración propia

\section{Conclusiones}

La empresa Caso de Estudio, es una Pymes, conforme las características establecidas por la Superintendencia de Compañías en el Ecuador. La empresa durante los años 2014 al 2016 realizó préstamos bancarios, en vista de la falta de liquidez e inyección de capital de parte de los socios; lo anterior generó en su Estado de Resultados Integrales un gasto financiero que redujo la utilidad de la entidad, esta información se obtuvo de la Superintendencia en vista que los estados financieros son públicos y cualquier usuario puede tomar dicha información financiera.

Se revisaron dos teorías sobre estructura de financiamiento que realizan las empresas, la primera teoría fue la Pecking orden que establece de donde se puede financiar una entidad y su coste; siendo así se puede obtener recursos financieros de las propias utilidades del negocio, de préstamos a terceros y emisión de acciones. La segunda teoría fue la Trade Off que menciona que los gastos de interés provocan una reducción o ahorro de impuestos, en este caso en el impuesto a la renta. 
El costo de los préstamos realizados por la empresa Caso de Estudio, se estimó en un $18 \%$, tasa alta, respecto a otras formas de conseguir dinero que existe en el mercado, por ejemplo, ofrecer a los socios una tasa del $10 \%$ por un préstamo, ya que el sistema financiero reconoce tasas menores al 5\% cuando un cliente invierte con ellos, lo que resulta atractivo para alguien que tiene recursos. Otra estrategia es el uso del mercado de valores, a través de la herramienta RED, que busca que las pequeñas empresas puedan obtener dinero. Para futuras investigaciones se puede profundizar en el análisis de esta herramienta RED, especialmente si las empresas están utilizando este esquema de obtener recursos.

\section{Bibliografía}

Azofra, V., \& Fernandez, A. (1999). Las finanzas empresariales 40 años. Papeles de economia española, 122-144.

Banco Mundial. (2014). Estado del arte sobre problemáticas financieras. Revista Escuela Administración de Negocios, Julio - Diciembre 2018(85), 163-179. doi:doi.org/10.21158/01208160.n85.2018.2056

Bernal, C. (2010). Metodología de la Investigación Tercera Edición. En C. A. Bernal. Colombia: Pearson.

Bolsa de Quito. (2014). Ley del Mercado de Valores de Quito. Quito: Bolsa de Quito.

Bolsa de Valores de Guayaquil. (2014). Diccionario de Economía y Finanzas. Quito.

Castaño, C., \& Quecedo, R. (2002). Introducción de Metodología de Investigación Cualitativa. Revista Psicodidáctica, núm 14, 5-39.

Castro Fernández, S. J. (s.f de s.f de 2013). Managment Social del no Equilibrio. Complejidad y ciencias del comportamiento: aportes teóricos y metodológicos a la ciencia de la dirección. Obtenido de Repositorio del Rosario: http://repository.urosario.edu.co/handle/10336/4930

Fernández , M., Conde, M. d., \& Zuliani, G. D. (2004). Contrastación de la Teoría del "Pecking Order". Departamento de Economía Financiera y contabilidad, 3-4.

Ferraro, C., Goldstein, E., Zuleta, L. A., \& Garrido, C. (2011). Eliminando barreras: El financiamiento a las pymes en América Latina. Santiago de Chile: Naciones Unidas.

Gitman, L. J. (2015). Principios de Administración Financiera.

Goetz, J. P., \& LeCompte, M. D. (1988). Etnografia y diseño cualitativo en investigacion cualitativa. Morata.

Gómez J., L. G. (2008). La teoria del Pecking Order. Actualidad Empresarial, 4.

Greene, W. (2007). Econometryc analysis of Panel Data (Vol. 6th Ed. ( $3^{\circ}$ en español)). USA: Prentice Hall.

Junta de Regulación Financiera. (2016). Normas aplicables al Registro Especial Bursátil. Recuperado el 19 de marzo de 2018, de Resolución No. 210-2016: https://www.bolsadevaloresguayaquil.com/resoluciones/Resolucion210v.pdf

Lecuona, R. (Mayo de 2009). El financiamiento a las Pymes en México: La experiencia reciente. Economía UNAM, 6(17). Obtenido de http://www.scielo.org.mx/scielo.php?script=sci_arttext\&pid=S1665952X2009000200004 
Londoño, R., \& Sanchez, J. (2017). Aplicación del Costo de Capital en las pymes en Colombia: una revisión. Science of human action, 2(2). Obtenido de https://www.funlam.edu.co/revistas/index.php/SHA/article/view/2470/1886

Majluf, N. S., \& Myers, S. C. (1984). Financiamiento corporativo y decisiones de inversión cuando las empresas tienen información que los inversores no tienen. Journal of Financial Economics, vol 13, No. 2, 187-221.

Martinez, P. (2006). El método del estudio de caso. Estrategia metodológica de la investigación científica. Pensamiento \& Gestión.

Ministerio de industrias y productividad. (12 de Agosto de 2012). Alternativas de Financiamiento a través del Mercado de Valores para Pymes. Obtenido de http://www.industrias.gob.ec/wp-

content/uploads/downloads/2012/08/2012_Alternativas_Financiamiento_PYMES_merca do_Valores.pdf

Murray, F., \& Vidhan, K. (2003). Testing the Pecking Order Theory of Capital Structure. Journal of Financial Economics, 67(2), 217-248.

Nuñez, P. (s.f de noviembre de 2013). REVNI: Alternativa de financiamiento para pequeñas $y$ medianas empresa. Obtenido de Pontificia Universidad Católica del Ecuador: http://repositorio.puce.edu.ec/handle/22000/10316

Osano, H., \& Languitone, H. (2016). Factors influencing access to finance by SMEs in Mozambique: case of SMEs in Maputo central business district. Journal of Innovation and Entrepreneurship(13).

Quinteros, J. J., \& De Marco, M. (2014). Análisis de Balances Clínicas y sanatorios. Redalyc, 117.

Romero, F., Zuray, M., \& Mary, V.-C. (2015). Fracaso empresarial de las pequeñas y medianas empresas (pymes) en Colombia. Suma de Negocios, 6(13), 29-41. doi:https://doi.org/10.1016/j.sumneg.2015.08.003

Ross, J., Westerfield, R., \& Bradfort, J. (2012). Finanzas Corporativas (9na ed.). México D.F: McGraw Hill.

Salgado, R. F. (s.f de s.f de 2015). El mercado de valores como alternativa de financiamiento para Pymes a través del análisis del registro de valores no inscritos en bolsa (REVNI) período 2009-2013. Obtenido de Repositorio Insitucional de la Universidad de las Fuerzas Armadas ESPE: http://repositorio.espe.edu.ec/handle/21000/10278

Steinar, K. (2011). Las entrevistas en Investigación Cualitativa. En Las entrevistas en Investigación Cualitativa (pág. 200). Madrid: Ediciones Morata S.L.

Superintendencia de Bancos del Ecuador. (s.f de s.f de 2009). Glosario de términos financieros. Obtenido de Glosario de términos financieros: http://www.sbs.gob.ec:7778/practg/sbs_index?vp_art_id=70\&vp_tip=2

Walker, M. C. (2001). Contratos Bancarios I y II. Santa Fe: Centro de Publicaciones Universidad Nacional del Litoral.

Zambrano, V. S., \& Acuña, G. A. (2013). Teoria del pecking order versus la Teoría del Pecking off para la empresa CoServicios. Redalyc, 205-236. 